Orthopäde $2017 \cdot 46: 805-807$

https://doi.org/10.1007/s00132-017-3466-7

C) Springer Medizin Verlag GmbH 2017

CrossMark

\section{R. Becker' ${ }^{1}$ R. Seil ${ }^{2,3} \cdot$ S. Kopf ${ }^{1}$}

'Zentrum für Orthopädie und Unfallchirurgie, Hochschulklinikum Brandenburg, Medizinische Hochschule Theodor Fontane, Brandenburg an der Havel, Deutschland

${ }^{2}$ Orthopädische Chirurgie, Centre Hospitalier de Luxembourg - Clinique d'Eich, Luxemburg, Luxemburg ${ }^{3}$ Sports Medicine Research Laboratory, Luxembourg Institute of Health, Centre Médical Norbert Metz, Luxemburg, Luxemburg

\title{
Meniskustherapie heute
}

Das vorliegende Themenheft diskutiert verschiedene Aspekte zur Pathogenese, Diagnostik und Therapie von Meniskuspathologien. Die Behandlung von traumatischen Meniskusrissen und degenerativen Meniskusläsionen stellt nicht nur eine der häufigsten Therapien in der Orthopädie und Unfallchirurgie dar, sondern wurde in den vergangenen Jahren auch sehr kontrovers diskutiert. Die Ära der Meniskusresektion bzw. Teilresektion als alleinige Behandlungsoption zählt zur Vergangenheit. Während der Meniskus früher als Appendix des Kniegelenkes gewertet wurde, ist seine protektive Funktion für das Kniegelenk heute unumstritten [5, 10]. Zusammenhänge zwischen Meniskusläsionen und der Arthroseentstehung werden ausführlich in dieser Ausgabe von Goebel et al. diskutiert [7]. Die enge Beziehung zwischen der Meniskusläsion und der Arthrosegenese basieren auf biomechanischen, neuromuskulären und molekularen Mechanismen. Biomechanische Arbeiten zeigten, dass der mediale und laterale Meniskus mindestens $50 \%$ der Lastübertragung im Kniegelenk übernehmen [1]. Die Entfernung des Meniskus bewirkt eine erhöhte Belastung des femorotibialen Knorpels. Die neuromuskuläre Aktivierung der Quadrizepsmuskulatur reduziert sich nach partieller Meniskusresektion [4]. Bisher bleibt ungeklärt, ob diese neuromuskulären Veränderungen durch die partielle Meniskusresektion bedingt sind oder ob sich das Gelenk zum Zeitpunkt der Operation bereits in einem präarthrotischen Zustand befindet. Während Meniskusläsionen einerseits primär eine arthrosefördernde Kaskade der Arthrosegenese auszulösen scheinen, stellen sie andererseits durch bereits bestehende Knorpelschäden eine sekundäre Pathologie dar [6].

Neben den klassischen Meniskusläsionen, vorrangig im Bereich des Hinterhorns und der Pars intermedia, werden Wurzel- und Rampenläsionen eine zunehmende Bedeutung beigemessen. Jahrelang wurden Wurzel- oder Rampenläsionen wenig Beachtung geschenkt. Traumatische Risse an der posterioren Wurzel des Außenmeniskus finden sich besonders häufig im Zusammenhang mit vorderen Kreuzbandrupturen [2]. Diese Risse können zu einem kompletten Funktionsverlust des lateralen Meniskus führen, besonders dann, wenn die meniskofemoralen Bänder, welche als sekundäre Stabilisatoren fungieren, fehlen. Die Meniskusrisse sollten im Rahmen der vorderen Kreuzbandplastik operativ versorgt werden [8]. Degenerative Wurzelläsionen bei bereits leicht arthrotisch verändertem Gelenk sollten gleichfalls operativ behandelt werden, auch wenn hier die Heilungschancen geringer sind und maßgeblich vom Grad der Gonarthrose abhängen.

Rampenläsionen entstehen am Übergang des Meniskushinterhorns zur Tibia. Sie werden leicht beim arthroskopischen Standardrundgang übersehen, da sie in der Regel nur über die posterioren Portale bzw. beim Durchgehen mit dem Arthroskop unter das hinter Kreuzband in den posteromedialen Recessus identifiziert werden können. Aus diesem Grund wird die Inzidenz dieser Meniskusläsionen im klinischen Alltag eher unterschätzt [9].

Obwohl Patienten in der Regel mit einer partiellen Meniskusresektion erfolgreich therapiert werden, zeigen sich die Konsequenzen erst nach vielen Jah- 
ren. Arthrotische Beschwerden führen $\mathrm{zu}$ einschneidenden Veränderungen in der Aktivität im Alltag und es können biologische, rekonstruktive oder gelenkersetzende Behandlungsmaßnahmen erforderlich werden.

Aufgrund der verschiedensten Meniskuspathologien und in engem $\mathrm{Zu}$ sammenhang mit der individuell unterschiedlichen Knorpel- und Bandsituation des Kniegelenkes sind entsprechende Behandlungskonzepte heutzutage sehr differenziert zu wählen.

Die Leitlinien zur Behandlung von Meniskuspathologie der Arbeitsgemeinschaft der Wissenschaftlichen Medizinischen Fachgesellschaften (AWMF) sind eine wesentliche Hilfe für die Therapieentscheidung (http://www.awmf.org/ leitlinien/detail/ll/033-006.html).

\section{1) Es wurde bewusst der Begriff der „Empfehlung" gewählt}

Die europäische Konsensempfehlung der European Society of Sports Traumatology, Knee Surgery and Arthroscopy (ESSKA) hat ebenfalls eine Empfehlung erarbeitet, die sich zum einen auf evidenzbasierte Untersuchungen und zum anderen auf die klinischen Erfahrungen von Kniespezialisten aus 27 europäischen Ländern stützt [3]. Aufgrund der Diskrepanz zwischen der evidenzbasierten Medizin und den klinischen Erfahrungen war eine umfangreiche Analyse der Therapieoptionen bezüglich der Behandlung von Meniskuspathologien erforderlich. Schlussfolgernd wurde festgestellt, dass degenerative Meniskusläsionen nicht zwingend eine sofortige operative Behandlung benötigen, denn auch eine konservative Therapie kann zum Erfolg führen. Selbst Konsensempfehlungen besitzen Grenzen, wie die Erfahrungen der europäischen Konsensgruppe der ESSKA zeigten. Es wurde bewusst der Begriff der „Empfehlung“ von dieser Gruppe gewählt, da keine ausreichende Evidenz besteht, um mehr als Empfehlungen geben zu können. Ungeachtet dessen muss die Therapieentscheidung aber in den Händen des behandelnden Arztes verbleiben, denn nur dieser kann bei dem jeweiligen Patienten sowohl das Beschwerdebild, die alltägliche
Einschränkung in der Aktivität, die Anamnesedauer, die Art der Meniskusläsion anhand der Bildgebung und den Grad der degenerativen Veränderung des Gelenkes gleichzeitig beurteilen und werten, um daraus eine Behandlung abzuleiten.

Verallgemeinerungen von Therapiekonzepten in der Medizin können dazu führen, dass die individuelle Behandlung des Patienten in den Hintergrund tritt. In Bezug auf die klinische Forschung drängen sich diesbezüglich eine Reihe von Fragen auf, die weiterer wissenschaftlicher Bearbeitung bedürfen: Welche Validität besitzen klinische Daten, wie wurden die Daten erhoben, welche Patienten wurden eingeschlossen und wie gut sind unsere Instrumente bezüglich einer klinischen Evaluierung? Diese Aspekte zeigen die Komplexität und den hohen Anspruch, der an die klinische Forschung gestellt wird.

Sollte der stetige Wissenszuwachs in der Medizin zu einer Verallgemeinerung der Therapiekonzepte genutzt werden oder sollte dieser eher zur Individualisierung der Therapie unserer Patienten führen?

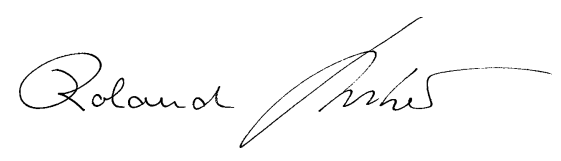

R. Becker

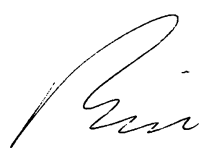

R. Seil

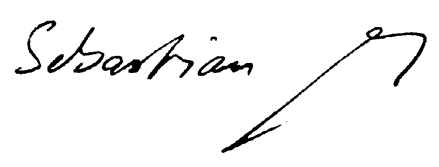

S. Kopf

\section{Korrespondenzadresse}

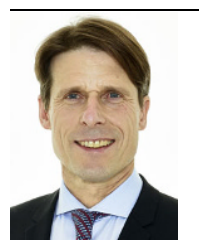

Prof. Dr. R. Becker

Zentrum für Orthopädie und Unfallchirurgie,

Hochschulklinikum

Brandenburg, Medizinische

Hochschule Theodor Fontane Hochstraße 26, 14770 Brandenburg an der Havel,

Deutschland r.becker@klinikumbrandenburg.de

Interessenkonflikt. R. Becker, R. Seil und S. Kopf geben an, dass kein Interessenkonflikt besteht.

\section{Literatur}

1. Aagaard H, Verdonk R (1999) Function of the normal meniscus and consequences of meniscal resection. Scand J Med Sci Sports 9:134-140

2. Bao HR, Zhu D, Gong H, Gu GS (2013) The effect of complete radial lateral meniscus posterior root tear on the knee contact mechanics: a finite element analysis. JOrthop Sci 18(2):256-263

3. Beaufils $P$, Becker R, Kopf $S$, Englund M, Verdonk R, Ollivier M, Seil R (2017) Surgical management of degenerative meniscus lesions: the 2016ESSKA meniscus consensus. Knee Surg Sports Traumatol Arthrosc 25(2):333-334

4. Becker R, Awiszus F (2001) Physiological alterations of maximal voluntary quadriceps activation by changes of knee joint angle. Muscle Nerve 24:667-672

5. Englund M (2008) The role of the meniscus in osteoarthritis genesis. Rheum Dis Clin North Am 34:573-579

6. Englund M, Guermazi A, Lohmander SL (2009) The role of the meniscus in knee osteoarthritis: a cause or consequence? Radiol Clin North Am 47:703-712

7. Goebel L, Reinhard J, Madry H (2017) Meniskusläsion. Präarthrotischer Zustand des Kniegelenks. Orthopäde. https://doi.org/10.1007/s00132-0173462-y

8. Kopf S, Stärke C, Becker R (2017) Meniskuswurzelläsionen: Klinische Relevanz und Therapie. Orthopäde. https://doi.org/10.1007/s00132-0173460-0

9. Seil R, Hoffmann A, Scheffler S (2017) Rampenläsionen. Tipps und Tricks in Diagnostikund Therapie Orthopäde. https://doi.org/10.1007/s00132-0173461-z

10. Verdonk R, Madry H, Shabshin N, Dirisamer F, Peretti GM, Pujol N, Spalding T, Verdonk P, Seil R, Condello V, Di Matteo B, Zellner J, Angele P (2016) The role of meniscal tissue in joint protection in early osteoarthritis. Knee Surg Sports Traumatol Arthrosc 24:1763-1774 
Hier steht eine Anzeige.

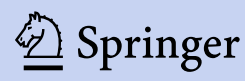

